\title{
Ilustrando a Segunda Lei de Newton no Século XXI
}

\author{
Illustrating Newton's Second Law in the $21^{\text {st }}$ Century \\ E. A. Veit e P. M. Mors \\ E-mail: eav@if.ufrgs.bremors@if.ufrgs.br \\ Instituto de Física \\ Universidade Federal do Rio Grande do Sul \\ Cx. P. 15051, 91051-970, Porto Alegre, RS, Brasil \\ V. D. Teodoro \\ E-mail:vdt@mail.fct.unl.pt \\ Faculdade de Ciências e Tecnologia \\ Universidade Nova de Lisboa, \\ 2825 Campus de Caparica, Portugal
}

Recebido em 1 de abril, 2002. Aceito em 6 de maio, 2002.

\begin{abstract}
Apresenta-se uma forma de ensinar a segunda Lei de Newton usando-se a modelagem computacional, de modo que, além das tradicionais situações lineares, problemas mais reais e atuais possam ser tratados. Ilustra-se com o aplicativo Modellus, que permite ao usuário (aluno ou professor) explorar modelos matemáticos baseados em equações diferenciais ou em funções. Para as situações físicas discutidas, são fornecidas as equações, parâmetros e condições iniciais necessárias, de modo que o leitor que opte por outro aplicativo tenha plenas condições de desenvolver as atividades propostas.

An approach to teach Newton's second Law is presented using computational modeling such that more real and contemporary problems may be treated, besides the traditional linear examples. This is illustrated with the software Modellus, which allows users (students or teachers) to explore mathematical models based on differential equations or on functions. The equations, parameters and initial conditions required to model each discussed situation are given, allowing the reader to implement the suggested activities even if he/she prefers to use some other software.
\end{abstract}

\section{Introdução}

A segunda Lei de Newton é, e entendemos que não deva deixar de ser, um dos focos centrais de qualquer curso introdutório de Mecânica; porém, se insistirmos em explorá-la somente de modo analítico, limitar-nosemos à Física de centenas de anos atrás. Aliás, talvez nem mesmo cheguemos a tanto, pois como os estudantes do ensino médio ou ingressantes no ensino universitário geralmente não têm conhecimentos para resolver uma equação diferencial, os problemas abordados costumam se limitar aos mais simples. Mesmo nestes casos, muitas vezes, é investido um longo tempo na solução matemática do problema, sem que seja dada a devida ênfase à situação física, relegada a um segundo plano.

Uma possibilidade de se dispor de mais tempo e de melhores condições para se concentrar nos aspectos físicos do problema, e não apenas na sua solução matemática, é recorrer ao computador, deixando que ele execute muitas das tarefas analíticas e numéricas. Entretanto, sendo a Matemática uma das linguagens mais importantes usadas na Física, claro está que aprender Física está, também, intimamente ligado com o aprendizado da Matemática. Então, a nossa proposta é que Física e Matemática sejam ensinadas de modo integrado [1]. Para isto, temos usado como ferramenta o aplicativo Modellus, concebido especialmente para modelagem computacional em Matemática e Ciências, que é distribuído livremente [2,3], e que é de fácil utilização mesmo por aprendizes sem conhecimento e destreza no uso de computadores [4].

A literatura de educação em Ciências está repleta de diferentes acepções para os termos modelo e modelagem [5]. Estamos interessados nos modelos físicos: descrições simplificadas e idealizadas de sistemas ou fenômenos físicos, aceitos pela comunidade de físicos, que envolvem elementos como representações (exter- 
nas), proposições semânticas e modelos matemáticos subjacentes; estes, passamos a denominar simplesmente de modelos [6]. No ensino/aprendizagem de Física, todos estes elementos são muito importantes, porém é a solução matemática que costuma consumir a maior parte do tempo e limitar as situações físicas discutidas. Entendemos que a modelagem computacional, ou seja, aquela em que o computador é utilizado para a solução dos modelos, é uma ferramenta excelente para enriquecer e atualizar o ensino de Física, propiciando o desenvolvimento de competências e habilidades preconizadas pelos Parâmetros Curriculares Nacionais para o Ensino Médio $[7,8]$.

Há diversos aplicativos desenvolvidos especialmente para a modelagem. A opção pelo Modellus reside no fato de que este aplicativo dispensa o uso de qualquer linguagem ou metáfora computacional e, adicionalmente, o simbolismo matemático utilizado é idêntico ao de um manuscrito, inclusive quando se formula um problema com equações diferenciais. Assim, caso o aprendiz ainda não tenha maior familiaridade com Matemática, pode vir a adquiri-la na medida em que estuda Física com esta ferramenta. Porém, a idéia principal deste artigo, a de que modelagem computacional é uma ferramenta excelente para enriquecer e atualizar o ensino de Física, pode ser implementada com outros programas como planilhas eletrônicas, Matlab e Mathematica, se bem que com maiores dificuldades em termos de tratamento de modelos e de criação de representações, como animações e gráficos.

Especificamente, exemplificamos como a segunda Lei de Newton pode ser ensinada de modo abrangente, com a resolução já no primeiro semestre universitário de problemas mais reais e/ou atuais do que os tradicionalmente tratados, e permitindo maior tempo para a discussão e aprendizagem dos aspectos relacionados às proposições dos modelos físicos.

\section{Ilustrações de solução da se- gunda Lei de Newton com o Modellus}

A segunda Lei de Newton é, conceitualmente, a mais simples das três leis do movimento e seu equacionamento é imediato, tão logo se tenha identificado o sistema e sua vizinhança. Na solução de um problema de Dinâmica, a maior parte do tempo é consumida em sua solução matemática, não no seu equacionamento. Nesta seção, exemplificamos como a segunda Lei pode ser facilmente resolvida, numericamente, pelo Modellus.

Neste trabalho, usamos a segunda Lei na forma:

$$
\vec{F}_{\text {res }}=m \vec{a}
$$

Uma vez conhecidas as condições iniciais (posição e velocidade) e a resultante das forças, $\vec{F}_{\text {res }}$, sobre a partícula de massa $m$, a sua trajetória fica completamente determinada. Estamos interessados na determinação desta trajetória, com a partícula sujeita a diferentes forças, isto é, possuindo diferentes valores de aceleração. Começamos com uma única força constante, por ser a situação mais simples, podendo o estudante resolver o problema analiticamente para comparar com os resultados fornecidos pelo aplicativo.

\section{1 Força constante e força dependente da velocidade}

Um exemplo de solução da segunda Lei de Newton no Modellus, para um problema unidimensional, é apresentado na Fig. 1. O eixo $O y$ é escolhido apontando-se para cima; daí o sinal negativo na primeira linha para a componente escalar da força. As quatro equações que aparecem na janela Modelo têm o seguinte significado, a começar pela de cima:

a componente do peso que atua sobre a partícula segundo o eixo $O y$;

a componente da aceleração na direção do movimento, eixo $O y$, admitindo-se que a única força que atua sobre a partícula é o peso;

a relação entre $v_{y}$, componente da velocidade na direção $y$, e $a_{y}$, componente da aceleração na direção $y\left(v_{y}\right.$ é calculado por integração a partir de $a_{y}$ );

a relação entre a posição $y$ e $v_{y}$ ( $y$ é calculado por integração a partir de $v_{y}$ ).

$\mathrm{Na}$ janela Condições Iniciais, é atribuído o valor de uma unidade à massa (1 kg, no Sistema Internacional) e 9,8 unidades à magnitude da aceleração da gravidade $\left(9,8 \mathrm{~m} / \mathrm{s}^{2}\right.$, no SI). Os valores iniciais para a posição e velocidade são nulos.

Deixando-se o tempo evoluir, Modellus resolve as equações diferenciais ${ }^{1}$, fornecendo para cada instante de tempo os valores numéricos de $a_{y}, v_{y}$ e $y$. Podemse, então, utilizar diferentes representações para se investigar o modelo em construção: tabelas, gráficos e a animação do movimento. Qualquer uma dessas possibilidades é implementada no Modellus com o uso do mouse, tão facilmente que mesmo estudantes que não tenham destreza no uso de computadores tornam-se capazes de construí-las ao final da primeira aula em que se valem deste aplicativo. Essas são as três opções apresentadas no menu Janela.

\footnotetext{
${ }^{1}$ Modellus resolve equações diferenciais utilizando o Método de Runge-Kutha de $4^{a}$ ordem. Os valores mínimo e máximo para o tempo, assim como o seu incremento, são escolhidos arbitrariamente pelo usuário. Por padrão, o valor mínimo é 0, o máximo 20, e o incremento ou passo 0,1 .
} 


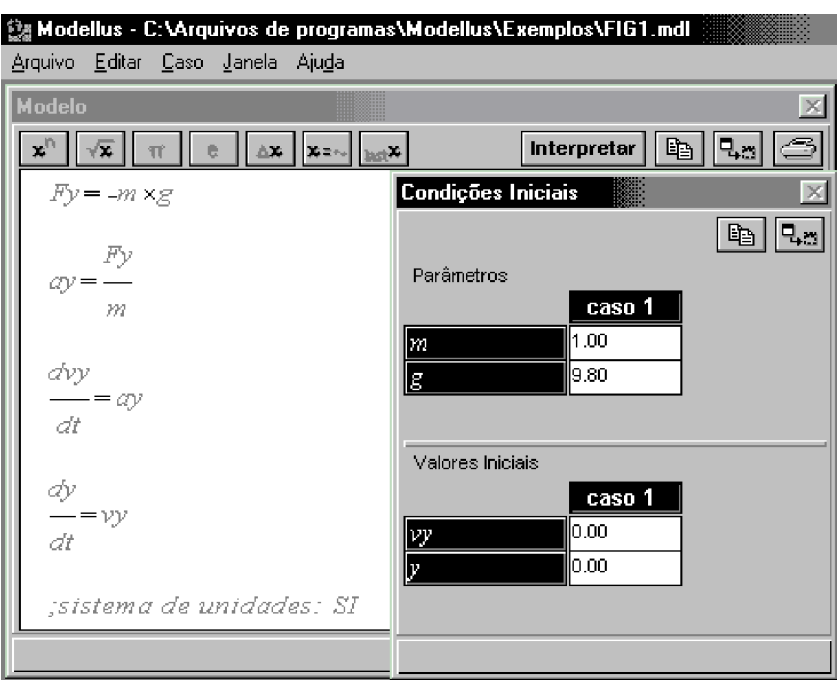

Figura 1. Equações referentes ao movimento unidimensional de uma partícula em queda livre, escritas no Modellus.

Variando-se os parâmetros $g$ e $m$ e os valores iniciais atribuídos à posição e à velocidade, o estudante pode investigar a influência dessas grandezas físicas sobre o movimento. Temos observado que, muitas vezes, somente neste estágio da aprendizagem é que os estudantes efetivamente começam a compreender o significado físico de algumas das variáveis utilizadas no equacionamento matemático do problema.

O leitor pode estar se questionando: e é justificável usar-se um computador para resolver o problema da partícula em queda livre? Entendemos que sim, pelos motivos que seguem.

As equações da Fig. 1 são equações freqüentes nos capítulos iniciais dos livros de Física Geral Universitária. Se o estudante não as compreende, o aplicativo pode servir como meio para conduzi-lo à sua compreensão. Por exemplo, ao se escrever

$$
\frac{d y}{d t}=\nu y
$$

e se pressionar o botão Interpretar na janela Modelo, imediatamente Modellus requererá que o aluno entre com $v_{y}$, como um parâmetro, e com o valor inicial para a posição $y$. Ou seja: ainda que o estudante não se dê conta de imediato que dado $v_{y}$, taxa de variação da posição com o tempo, só é possível conhecer-se a posição num instante qualquer, caso se conheça a posição no instante inicial, Modellus o lembrará disto. (Modellus é excelente para se introduzir os conceitos básicos de Cálculo Diferencial e Integral e de equações diferenciais, podendo tornar o ensino de Cinemática atrativo e instrutivo no exercício de cálculo de uma variável; mas isto foge ao escopo do presente trabalho.)

Pela primeira vez, o estudante recorre a equações diferenciais para equacionar um problema, e entendemos que ele deva fazê-lo começando pelos problemas mais simples. Modellus se encarregará de resolver as equações diferenciais.
O estudante estará, em poucos meses, aprendendo o conceito de derivada no seu curso de Cálculo Diferencial e Integral e é instrutivo que se vá familiarizando com o uso de derivadas.

A partir do modelo para a partícula sob a ação da força peso, preferentemente construído pelo aprendiz, a inclusão da resistência do ar resume-se à soma de algum termo à primeira linha da janela Modelo, e todo o restante não precisará ser modificado. Assim, pode-se discutir, já na segunda semana de aula do curso universitário, a solução do problema de uma queda que não é livre. $\mathrm{O}$ estudante pode experimentar como seria o movimento se à força se somasse um termo resistivo do tipo $b v_{y}$, simplesmente acrescentando este termo à força que atua sobre a partícula:

$$
F y=-m \times g-b \times \nu y
$$

Este último argumento é o que nos motivou a escrever o presente trabalho. Entendemos que não faz sentido, em pleno século XXI, limitar-se o estudo de Dinâmica aos problemas a que Galileu se dedicava. (E mesmo Galileu já se preocupava com a resistência do ar.) Muito mais importante do que chegar-se a uma solução exata de um problema aproximado, como é a queda livre, é questionar-se sobre os aspectos físicos relevantes, sobre as hipóteses assumidas, as aproximações empregadas, as limitações dos modelos, as grandezas físicas relevantes e as suas relações. Recorrendo-se ao computador para a solução do modelo matemático subjacente ao modelo físico, o aluno pode dispor de muito mais tempo para se concentrar nos aspectos físicos e no equacionamento dos problemas.

A extensão do caso unidimensional para o bidimensional é imediata: basta escrever as correspondentes expressões para a componente horizontal das quantidades físicas em questão (força, aceleração, velocidade e posição). Com mais quatro linhas na janela Modelo (Fig. 2) pode-se descrever o movimento parabólico de qualquer projétil sob ação de uma força constante.

$$
\begin{gathered}
F x=0 \\
a x=\frac{F x}{m} \\
\frac{d \nu x}{d t}=a x \\
\frac{d x}{d t}=\nu x
\end{gathered}
$$

Figura 2. Equações do movimento para a componente horizontal $(x)$ de uma partícula em queda livre escritas no Modellus.

A animação do movimento é obtida colocando-se um objeto, uma bola, por exemplo, na posição desejada da tela e especificando-se as suas coordenadas horizontal e vertical como sendo as coordenadas $(x, y)$, soluções das equações diferenciais das Figs. 1 e 2 . À 
medida que o tempo evolui, a bola move-se na tela. A Fig. 3 ilustra uma animação em que, além da trajetória do projétil, aparece uma imagem estroboscópica mostrando as posições sucessivas ocupadas pelo projétil de tempos em tempos iguais.

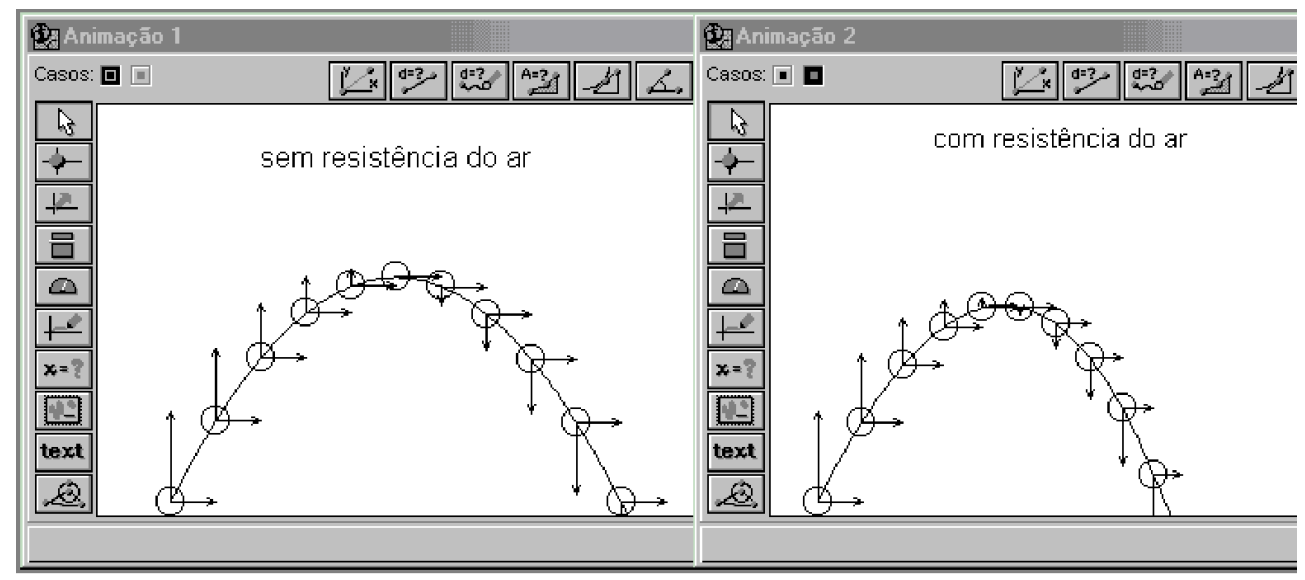

Figura 3. Movimento de um projétil, (a) sem e (b) com resistência do ar.

Também estão indicadas as componentes horizontal e vertical da velocidade. Isso é feito unindo-se ao projétil um vetor cujas componentes escalares coincidem com os valores obtidos para $v_{x}$ e $v_{y}$. Discussões a respeito da nulidade da componente $y$ da velocidade no ponto mais alto da trajetória, mas não da componente $x$, e, especialmente, as motivadas por variações no valor do parâmetro $b$, são oportunas.

\section{2 Movimento oscilatório unidimen- sional}

Para investigar um sistema massa-mola - oscilador unidimensional, em que a resultante das forças tem a direção do eixo $O x$ - basta substituir, no conjunto de equações apresentado na Fig. 2, a componente da força na direção $x$ por

$$
F x=-k \times x
$$

onde $k$ representa a constante elástica da mola. A componente vertical da posição, $y$, é mantida constante.

Ao pressionar o botão Interpretar, a massa da partícula e a constante elástica da mola são identificadas como parâmetros, enquanto para os valores iniciais são requeridas posição e velocidade ${ }^{2}$. A Fig. 4 ilustra uma animação do movimento e os correspondentes gráficos para as componentes escalares de posição, velocidade e aceleração ${ }^{3}$.

Neste caso, o estudante trabalha com um modelo cuja solução matemática é incapaz de obter, mas isso não o impede de entender a evolução dinâmica do sistema, as variáveis relevantes e suas relações. Ao invés de se provar analiticamente que a posição do oscilador pode ser expressa por uma senóide, uma linha adicional no modelo do tipo:

$$
x 1=A \times \cos (w \times t+\text { delta })
$$

permite comparar $x 1$, numericamente ou graficamente, com a solução $x$ obtida via equação diferencial. A comparação auxiliará o estudante na compreensão das quantidades físicas que caracterizam o movimento oscilatório como a amplitude, $A$, a freqüência angular, $w$, e a constante de fase, delta. A propósito, a observação do movimento de uma partícula cuja posição é dada pela equação 4 para diferentes valores de delta, escolhidos arbitrariamente pelo próprio estudante, além de elemento motivador, facilita a aprendizagem do significado da constante de fase, e da sua relação com a posição e a velocidade iniciais do sistema.

Efeitos de ressonância podem ser investigados com a inclusão de uma força de excitação externa, caracterizada por uma freqüência de oscilação $w_{-} e x t e$ uma intensidade máxima $f$ :

$$
F x=-k \times x-b \times \nu x+f \times \cos \left(w_{-} e x t \times t\right)
$$

\footnotetext{
${ }^{2}$ É comum, numa primeira tentativa, o estudante não alterar o valor zero atribuído, por padrão, tanto à posição quanto à velocidade iniciais da massa presa à mola, e ficar surpreso porque a massa não se move.

${ }^{3}$ A janela Condições Iniciais permite que se introduzam até cinco conjuntos de valores (cinco casos), tornando-se muito prática a comparação de diferentes situações físicas descritas por um mesmo modelo. Neste exemplo, isso possibilitaria a investigação simultânea de vários tipos de movimento harmônico, amortecidos e não-amortecidos.
} 
sem amortecimento $(b=0)$
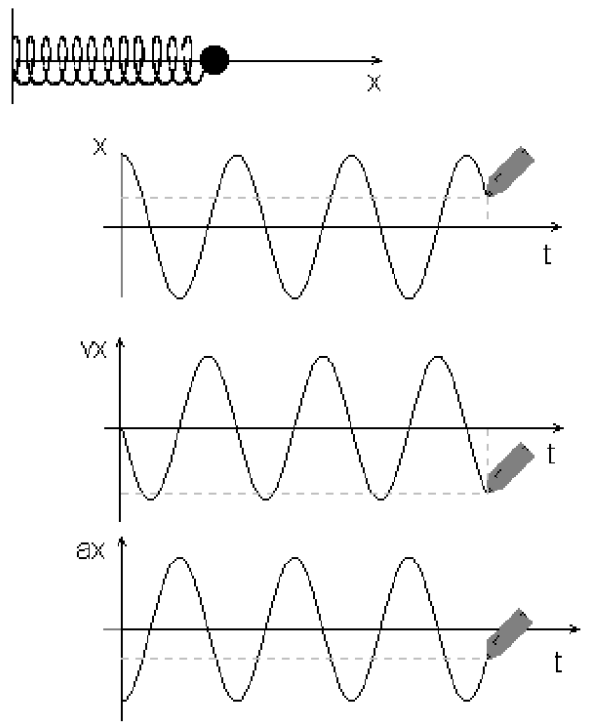

com amotecimento $(b+0)$
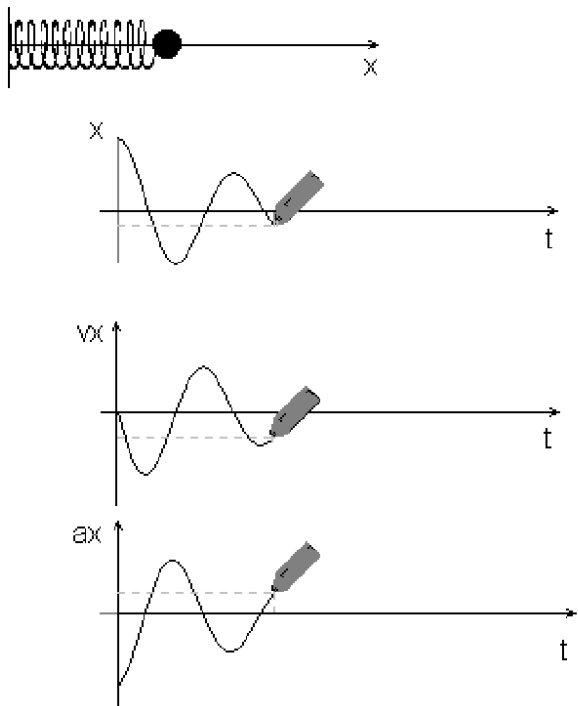

Figure 4. Sistema massa-mola (a) sem e (b) com amortecimento.

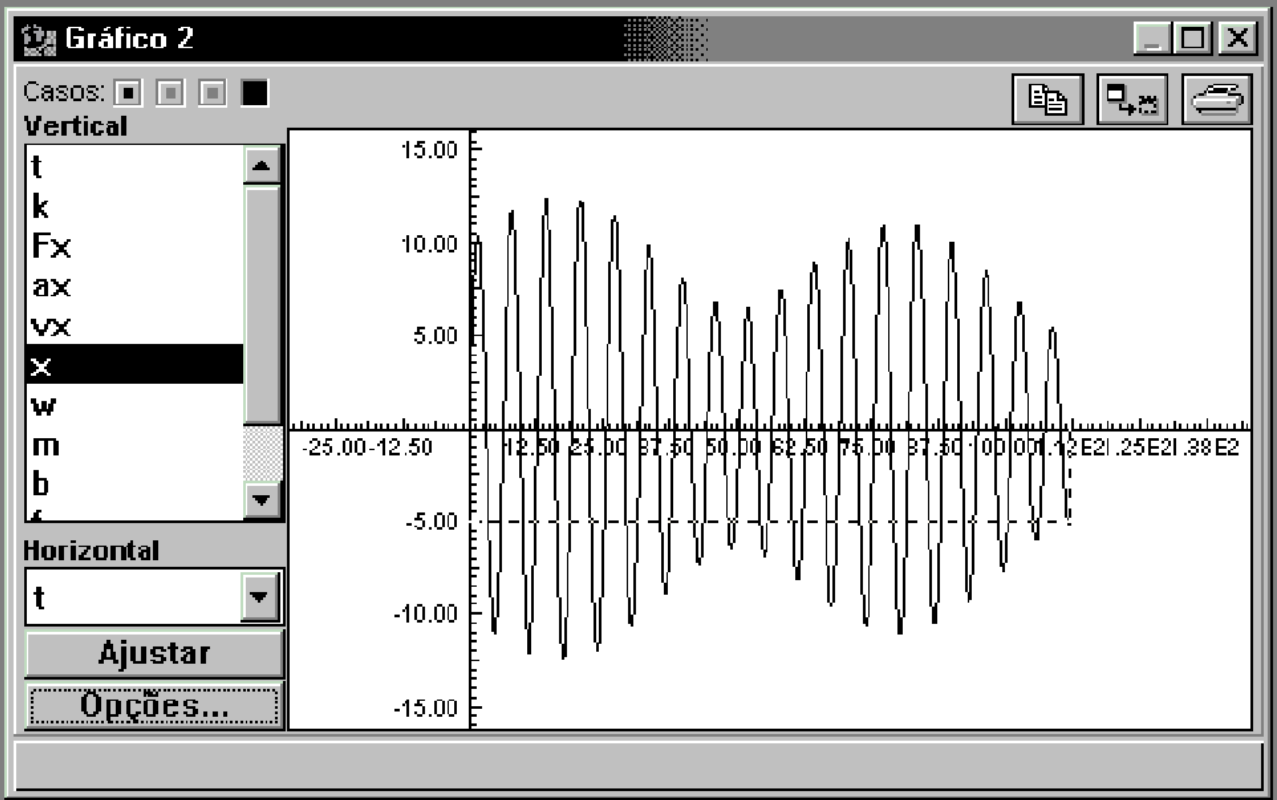

Figura 5. Amplitude de oscilação para uma freqüência externa (w_ext na equação 5) próxima da freqüência natural do oscilador.

Uma ilustração da amplitude de um oscilador sujeito a esta força é vista na Fig. 5 .

\section{II.3 Pêndulo físico}

A obtenção das equações de movimento para um pêndulo (físico ou simples) é facilitada aplicando-se a versão rotacional da segunda Lei de Newton:

$$
\vec{\tau}_{R o}=I_{0} \vec{\alpha},
$$

sendo $\vec{\tau}_{R o}$ a resultante dos torques em relação a um ponto $o ; I_{o}$, o momento de inércia do sistema em relação a um eixo que passa por este ponto e tem a direção do torque; e $\vec{\alpha}$, a aceleração angular do sistema.

Um exemplo típico da aplicação da versão rotacional da segunda Lei de Newton é o pêndulo físico (um corpo rígido), cujo modelo se encontra na Fig. 6. Considerase o eixo $O x$ apontando para a direita, $O y$ para cima e $O z$, para fora da página ${ }^{4}$ (ou da tela do computador),

\footnotetext{
${ }^{4}$ Ao longo deste trabalho, adota-se este sistema de coordenadas.
} 
perpendicularmente ao plano de oscilação, definido por $O x$ e $O y$. Os parâmetros deste modelo são:

- $m$, massa do pêndulo;

- g, a magnitude da aceleração da gravidade;

- $I_{o}$, momento de inércia em relação ao centro de massa;

- $D$, distância do centro de massa ao centro de oscilação, situado na origem do sistema de coordenadas adotado.

As sete equações que aparecem na Fig.6 representam, respectivamente:

- $I_{o}$, momento de inércia em relação à origem, obtido pelo teorema dos eixos paralelos;

- o torque (restaurador) exercido pela força peso, admitida como a única força que exerce torque, em relação à origem do sistema de coordenadas;

- a equação 6 , projetada no eixo $O z$;

- a relação entre $w$, componente da velocidade angular na direção $z$, , e $\alpha$, componente da aceleração angular na direção $z$;

- a relação entre a posição angular $\theta$ e $w$;

- as coordenadas retangulares, $x$ e $y$, da posição do centro de massa do corpo rígido, em função das suas coordenadas polares.

Para solução dessas equações diferenciais, automaticamente Modellus requer os valores iniciais para a posição angular e velocidade angular.

$$
\begin{aligned}
& I o=I c m+m \times D^{2} \\
& a \operatorname{loh} \alpha=-\frac{\operatorname{torg} L e}{\operatorname{To}} \\
& \frac{d o m e g a}{d t}=\operatorname{coh} \alpha \\
& \text { dheta } \\
& a^{t}=o m e g a \\
& x=D \times \sin (t h e t a) \\
& y=-D \times \cos (t h e t a)
\end{aligned}
$$$$
\operatorname{torg} 2 \theta=-m \times g \times D \times \sin (t h e t c)
$$

Figura 6. Equações referentes ao movimento de um pêndulo físico, escritas no Modellus.

As componentes retangulares são obtidas para possibilitar a animação do movimento. (O sistema de coordenadas adotado é o retangular, sendo necessário transformar coordenadas polares para as retangulares, caso se queira observar o movimento do pêndulo físico.)
Os argumentos apresentados na seção II.1, em favor do uso do computador em problemas que envolvem força constante, são aqui ainda mais justificáveis, pois os estudantes têm menor familiaridade com as grandezas angulares do que com as lineares. Ademais, a fim de obter-se uma solução analítica para este problema, é necessário proceder-se à aproximação linear, tomando-se

$$
\operatorname{sen}(\theta) \approx \theta
$$

e limitando-se a descrição do movimento a pequenas amplitudes.

Apregoamos o uso do computador na solução deste problema, também porque se pode:

- construir (rapidamente) uma tabela que ilustre a equação 7, dando elementos numéricos para a discussão do limite de validade da aproximação;

- considerar qualquer amplitude de oscilação. A comparação da solução numérica com a analítica, para uma amplitude de oscilação qualquer, enriquece a discussão do que significam pequenas amplitudes e do limite de validade da aproximação linear;

- investigar a variação do período com a amplitude; - incluir resistência;

- incluir excitação externa, possibilitando ilustrar ressonância e, particularmente, caos;

- estudar física não-linear.

Uma ilustração simples de assunto atual - regimes caóticos - é a da Fig. 7, que mostra o modelo do pêndulo sujeito a uma excitação externa oscilante, tratado na referência [9]. A equação de movimento para o sistema é:

$$
\frac{d \omega}{d t}=-\frac{1}{q} \omega-\operatorname{sen} \theta+p \cos \phi
$$

onde $\theta$ é a posição angular do pêndulo, medida com relação à vertical, e

$$
\omega=\frac{d \theta}{d t}
$$

é sua velocidade angular. A fase $\phi$ é tal que sua variação temporal é a medida da freqüência angular forçada, $\omega_{D}$, imposta por algum agente externo no ponto de suspensão:

$$
\omega_{D}=\frac{d \phi}{d t}
$$

O termo forçado tem a amplitude $p$, e o parâmetro de amortecimento é dado por $q$. Foram utilizados os valores $q=2$ e $\omega_{D}=2 / 3$. (É adotado um sistema de unidades conveniente, com o tempo adimensional, mas não há por que se deter neste aspecto, para a compreensão qualitativa do que se deseja ilustrar.).

A equação de movimento deste sistema bidimensional é não-linear. Sistemas não-lineares podem apresentar a característica de terem seu desenvolvimento 
temporal fortemente dependente das condições iniciais. No exemplo da Fig. 7, dois pêndulos regidos pela equação 8 são postos em movimento, ambos com o mesmo parâmetro $p(p=1)$, mesmas condições iniciais $\omega_{0}=1$ rads e $\phi_{0}=0,9 \mathrm{rad}$, e com posições angulares iniciais diferindo em $0,05 \%: \theta_{0}=2 \mathrm{rad}$ e $2,01 \mathrm{rad}$, respectivamente. Posta a funcionar, a animação mostra os dois pêndulos oscilando de formas visualmente difíceis de distinguir, também não havendo praticamente distinção entre os diagramas de fase, traçados no gráfico $\omega$ versus $\theta$. Alterando-se o parâmetro $p$, nos dois pêndulos, para o valor $p=1,5$, as diferenças passam a ser evidentes, tanto na observação das trajetórias no espaço de configuração, quanto no espaço de fase. Isto significa que o novo valor do parâmetro $p$ pertence à região de valores em que o sistema apresenta comportamento caótico, manifestando esta forte dependência com as condições iniciais.

Esta é a Física dos nossos dias, Física Contem- porânea, que deve ser levada à discussão no primeiro semestre de um curso universitário e/ou no nível médio e não somente numa disciplina específica de final de alguns poucos cursos universitários.

\section{II.4 Movimento planetário}

A obtenção analítica da trajetória de duas partículas que interagem gravitacionalmente foge ao escopo de um curso introdutório de mecânica; porém sua resolução numérica é simples e deve ser utilizada para ilustrar, por exemplo, as possíveis trajetórias (curvas cônicas).

Basta que seja introduzida a força gravitacional entre duas partículas:

$$
\vec{F}=-G \frac{m_{1} m_{2}}{r^{2}} \hat{e}_{r}
$$

para que se possa discutir a situação dinâmica do sistema de duas partículas.

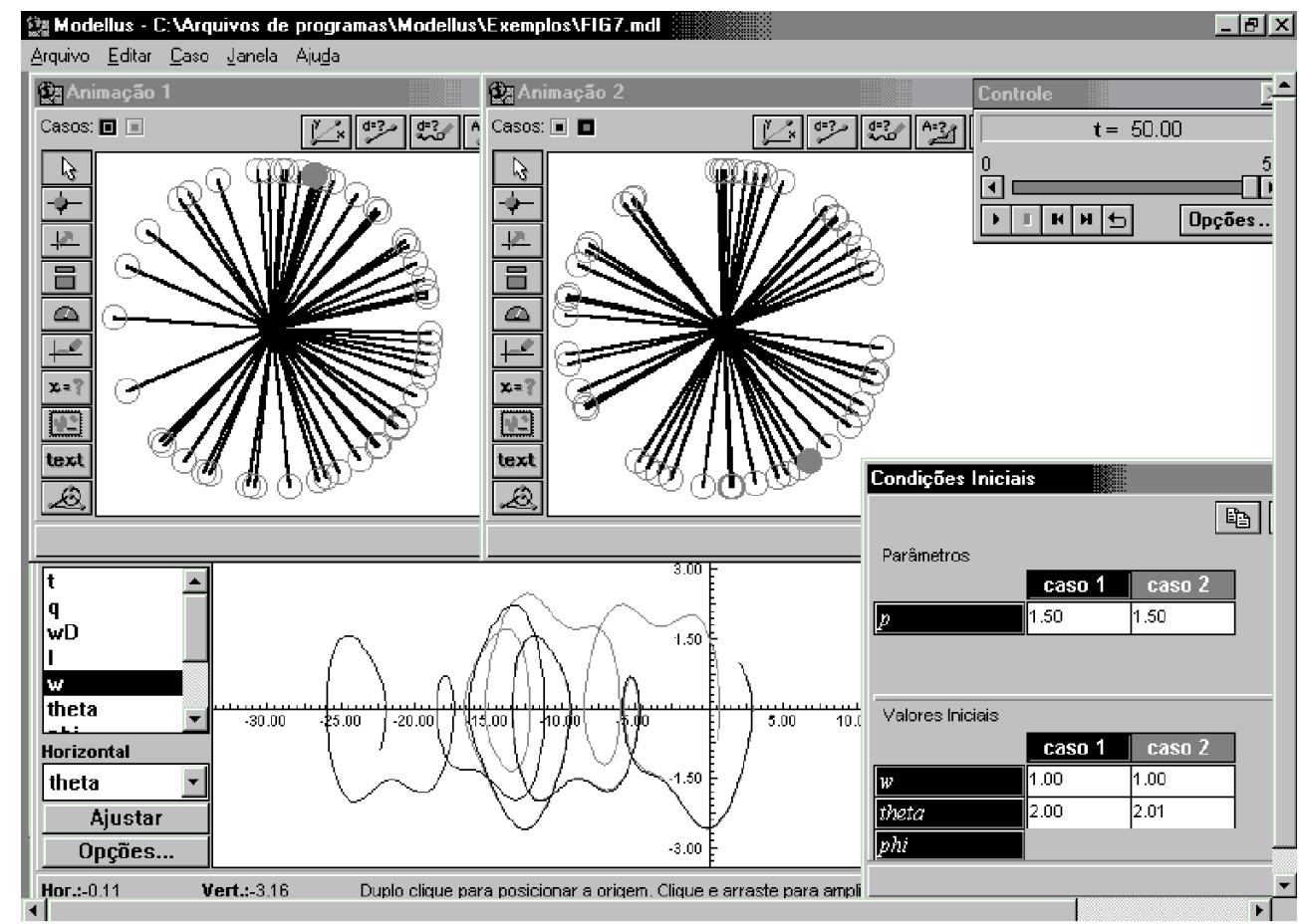

Figura 7: Regime caótico apresentado por um pêndulo simples sujeito a excitação externa [1]. Os dois pêndulos são soltos de posições angulares levemente diferentes (2,00 rad e 2,01 rad) e apresentam movimentos bem distintos em tempos subseqüentes, porque os parâmetros adotados se encontram na região do regime caótico. A variação de parâmetros permite observar que em outras regiões este comportamento não se verifica.

Consideremos, inicialmente, a interação entre duas partículas de massas $m_{1}$ e $m_{2}$, sendo $m_{1}>>m_{2}$. Neste caso, o recuo da massa $m_{1}$ é desprezível, e concentremonos apenas na trajetória da partícula de menor massa. Para simplificar, tomemos um sistema de coordenadas cuja origem coincide com a posição de $m_{1}$.

Os parâmetros necessários são:

- $m_{1}$ e $m_{2}$, massas das duas partículas que interagem;
- $G$, constante de gravitação.

A trajetória da partícula $m_{2}$ pode ser obtida no Modellus com as dez equações da Fig. 8:

- $r$, distância entre as partículas $m_{1}$ e $m_{2}$, escrita em termos das coordenadas $(x, y)$ de $m_{2}$;

- F, magnitude da força de atração gravitacional entre ambas as partículas (equação 9);

- $F_{x}$ e $F_{y}$, componentes horizontal e vertical da força que atua sobre $m_{2}$; 
- $a_{x}$ e $a_{y}$, componentes da aceleração de $m_{2}$ obtidas de $F_{x}$ e $F_{y}$, respectivamente;

- $v_{x}$ e $v_{y}$, componentes da velocidade de $m_{2}$ nas direções $x$ e $y$ obtidas de $a_{x}$ e $a_{y}$, respectivamente;

- $x$ e $y$, componentes da posição de $m_{2}$ nas direções $x$ e $y$ obtidas de $v_{x}$ e $v_{y}$, respectivamente.

Ao interpretar o modelo, Modellus automaticamente requer os valores iniciais para a posição $(x, y)$ e velocidade $\left(v_{x}, v_{y}\right)$ da massa $m_{2}$. Um gráfico de $y$ versus $x$ permitirá investigar diferentes trajetórias.

$$
\begin{array}{cl}
r=\sqrt{\left(x^{2}+y^{2}\right)} & a x=\frac{F x}{m 2} \\
F=G \times \frac{m I \times m 2}{r^{2}} & \frac{\omega y}{m y} \\
F x=-F \times \frac{m 2}{r} & \frac{d x}{d t}=a x \\
F y=-F \times \frac{d v y}{r} & \frac{d y}{d t}=a y \\
& \frac{d x}{d t}=v x \\
& \frac{d y}{d t}=v y
\end{array}
$$

Figura 8: Equações para o movimento de uma partícula de massa $m_{2}$ que interage gravitacionalmente com outra de massa $m_{1}>>m_{2}$.

A investigação de um sistema em que ambas as partículas se movem requer um pouco mais de geometria. A distância entre as duas partículas, $r_{12}$, é obtida de $\left(x_{1}, y_{1}\right)$ e $\left(x_{2}, y_{2}\right)$, coordenadas das massas $m_{1}$ e $m_{2}$ :

$$
r 12=\sqrt{\left(\left(x_{1}-x_{2}\right)^{2}+\left(y_{1}-y_{2}\right)^{2}\right)}
$$

Esta é a distância relevante no cálculo da força gravitacional, cujas componentes $x$ e $y$, para uma das partículas, são:

$$
\begin{aligned}
& F x=-F \times \frac{x_{1}-x_{2}}{r 12} \\
& F y=-F \times \frac{y_{1}-y_{2}}{r 12}
\end{aligned}
$$

A obtenção das coordenadas de uma das partículas é feita como no modelo da Fig. 8. A da outra partícula pode ser obtida através da coordenada do centro de massa que, estando o sistema isolado, poderá ser tomado em repouso.

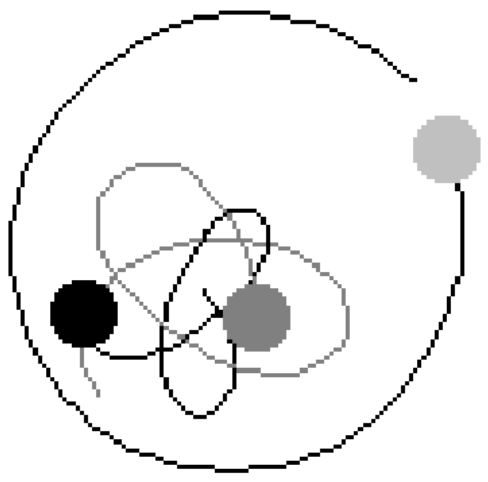

Figura 9: Visualização de um sistema de três massas que interagem gravitacionalmente, construída com o Modellus.

Imediata e interessante seria a generalização para forças do tipo $r^{x}$ em que $x$ é um expoente qualquer e as trajetórias deixam de ser as curvas cônicas. Também a interação gravitacional entre três partículas, visualizada na Fig. 9, pode ser construída por um estudante suficientemente instigado.

\section{Comentários finais e con- clusões}

Neste trabalho, concentramo-nos na possibilidade e necessidade de enriquecimento das aulas de Física através da modelagem computacional, deixando de enfocar as aulas experimentais que precisariam estar acopladas à discussão da segunda Lei de Newton. Deixemos, pelo menos, o alerta de que nossos laboratórios didáticos de Física, tanto universitários quanto escolares, estão, em sua quase totalidade, completamente obsoletos, não incorporando o computador como instrumento de medida e análise. O uso de microcomputadores em aquisição e visualização de dados em tempo real é um elemento dos laboratórios didáticos de países avançados há uma década [10-11], enquanto no Brasil está limitado a poucos estabelecimentos de ensino. Acreditamos que esta lacuna poderá ser preenchida a curto prazo com a implementação de aquisição automática via placa de som, dispensando interfaces externas [12-14]. Especificamente em relação à segunda Lei, a observação em tempo real, por exemplo, da variação da posição em função do tempo de um pêndulo $[12,15]$ possibilita o estudo de movimento amortecido com muito mais detalhes além dos usualmente observados. Também neste caso, a modelagem computacional se torna útil para a 
avaliação dos modelos construídos na tentativa de descrição dos dados.

Defendemos que a modelagem computacional é uma ferramenta indispensável para o ensino de Física nos dias atuais, devendo ser usada como recurso metodológico para promover a interação pessoal e a troca de significados através das atividades de grupo, em que a discussão, a conjetura e a avaliação de idéias são atividades dominantes. Nossa convicção, baseada na experiência em sala de aula, é compartilhada por diversos professores de ensino médio e universitário de diferentes países, que têm recorrido ao Modellus no ensino de Ciências e Matemática [16,17]. Estudos sistemáticos sobre a influência do uso de aplicativos de modelagem no processo de ensino/aprendizagem seriam muito bemvindos, tanto em relação ao Modellus, quanto a outros tantos aplicativos que têm sido utilizados em larga escala. Registramos, ainda, que abordagens à segunda Lei de Newton similares à nossa têm sido empregadas em várias universidades brasileiras; por exemplo, por Aguiar [18].

Para clareza deste artigo, fez-se necessário ilustrá-lo com algum aplicativo, e optamos pelo Modellus porque entendemos que é dos mais recomendáveis para um nível introdutório. Por vezes, apresentamos algumas características específicas do Modellus, para permitir que o leitor, se interessado, possa utilizar de imediato este aplicativo. Além disto, fornecemos as equações, parâmetros e condições iniciais necessárias, a fim de que o leitor que opte por outro aplicativo tenha plenas condições de desenvolver atividades em sala de aula no sentido de explorar a modelagem computacional no ensino de Física. Afinal, como dito por um de nós (VDT) "inteligência, emoção, cultura, poesia e arte residem no usuário, não no aplicativo".

\section{Agradecimentos}

Agradecemos aos professores J. Valadares, M. A. Moreira e V. H. Guimarães pela leitura crítica deste manuscrito.

\section{Referências}

[1] VEIT, E. A.; MORS, P. M. Física geral universitária: mecânica. Instituto de Física - UFRGS, 1999.

[2] TEODORO, V. D.; VIEIRA, J. P. D.; CLÉRIGO, F. C. Modellus 2.01: interactive modelling with mathematics. Monte Caparica: Faculdade de Ciência e Tecnologia Universidade Nova de Lisboa, 2000.

[3] TEODORO, V. D. Modellus: experiments with mathematical models. Disponível em:

$<$ http://phoenix.sce.fct.unl.pt/modellus/>. Acesso em: 20 março 2002 .
[4] TEODORO, V. D. From formulae to conceptual experiments: interactive modelling in the physical sciences and in mathematics. In: INTERNATIONAL CoLos CONFERENCE NEW NETWORK-BASED MEDIA IN EDUCATION, 1998, Maribor, Eslovênia. [S.l.: s.n.], 1998. p. 13-22.

[5] KRAPAS, S.; QUEIROZ, G.; COLINVAUX, D.; FRANCO, C. Modelos: uma análise de sentidos na literatura de pesquisa em ensino de ciências. Invest. Ens. Ci., Porto Alegre, v. 2, n. 3, p. 185-205, set./dez. 1997.

[6] GRECA, I. M.; MOREIRA, M. A. Mental, physical, and mathematical models in the teaching and learning of physics, Science Education, v. 86, p. 106-121, 2002.

[7] Brasil. Ministério da Educação, Secretaria de Educação Média e Tecnológica. Parâmetros curriculares nacionais: ensino médio. Brasília: MEC, 1999.

[8] VEIT, E. A. e TEODORO, V. D. Modelagem no ensino/aprendizagem de Física e os novos parâmetros curriculares nacionais para o ensino médio, Rev. Bras. Ens. Fís., São Paulo, aceito para publicação.

[9] BAKER, G. L. e GOLLUB, J. P. Chaotic Dynamics: an Introduction, Cambridge University Press, 1990.

[10] SCAIFE, J. Datalogging: where are we now? Physics Education, v. 28 n.2, 83-86, 1993.

[11] ROGERS, L., e WILD, P. Data-logging: Effects on practical science. Journal of Computer Assisted Learning, v.12 n.3, 130-145, 1996.

[12] HAAG, R. Utilizando a placa de som do micro PC no laboratório didático de Física. Rev. Bras. Ens. Fís, São Paulo, v. 23, n. 2, 176-183, junho 2001.

[13] MONTAROYOS E. e MAGNO, W. C. Aquisição de dados com a placa de som do computador, Rev. Bras. Ens. Fís., São Paulo, v. 23, n. 1, 57- 62, mar. 2001.

[14] AGUIAR. C. E. e LAUDARES, F. Aquisição de dados usando LOGO e a porta de jogos do PC. Rev. Bras. Ens. Fís, São Paulo, v. 23, n. 4, 371-380, dez. 2001.

[15] SOUZA, D. F. de, SARTORI, J., BELL, M. J. V. e NUNES, L. A. O. Aquisição de dados e aplicações simples usando a porta paralela do micro PC. Rev. Bras. Ens. Fís, São Paulo, v. 20, n. 4, 413-422, dez. 1998.

[16] BRIGHTON. European Commission. Science Teacher Training in an information Society. Annexe of case studies: annexe to the transversal report on WP 1.2. Brighton: Institute of Education, University of Sussex, 2000. P. 145-164.

[17] TEODORO, V. D. Teachers, Modelling and Modellus a questionnaire. Disponível em: <http://phoenix.sce.fct.unl.pt/modellus/ evaluations_and_comments / questionnaire_profs.htm $>$. Acesso em: 23 março 2002.

[18] AGUIAR, C. E. Notas de aula. Disponível em: $<$ http://omnis.if.ufrj.br/infoenci/notas.htm>. Acesso em: 14 abril 2002 